\title{
Carbon stars in populations of different metallicity
}

\author{
M.A.T. Groenewegen \\ Max-Planck-Institut für Astrophysik, Karl-Schwarzschild-Straße 1, \\ D-85740 Garching, Germany
}

\begin{abstract}
Our current knowledge of carbon stars in the Local Group and beyond, is discussed. Although many carbon stars and late M-stars have been identified in external galaxies, a coherent understanding in terms of the chemical evolution- and star-formation-rate history of a galaxy is still largely lacking. Issues that need to be addressed are: 1) for some of the larger galaxies only a small fraction in area has been surveyed so far, 2) surveys have been conducted using different techniques, and may be incomplete in bolometric magnitude, 3) only for some galaxies is there information about the late M-star population, 4) not all galaxies in the Local Group have been surveyed, 5) only for a sub-set of stars are bolometric magnitudes available.

From the existing observations one can derive the following: the formation of carbon stars is both a function of metallicity and star formation. In galaxies with a similar star-formation-rate history, there will be relatively more carbon stars formed in the system with the lower metallicity. On the other hand, the scarcity of AGB-type carbon stars in some systems with the lowest metallicity indicates that these galaxies have had a low, if any, star-formation-rate history over the last few Gyrs.
\end{abstract}

\section{Introduction}

Carbon stars are tracers of the intermediate age population in galaxies. Either they are currently undergoing third dredge-up (the cool and luminous N-type carbon stars), or have been enriched with carbon-rich material in a binary system when the present-day white dwarf was on the AGB (the carbon dwarfs, CH-stars. The R-stars may be the result of coalescing binaries [McClure 1997]).

Since their spectral signature is very different from the one of oxygen-rich stars, it is relatively easy to identify carbon stars even at large distances. In Sect. 2 the different techniques to identify carbon stars are discussed, and in Sect. 3 the various surveys for carbon stars in external galaxies are summarised. The results are discussed in Sect. 4.

\section{Methods}

Basically three methods have been used to identify carbon stars in external galaxies: (optical) spectroscopy of red stars, a combination of a grating and a prism (GRISM), and narrow-band filters. The first method was used in the "early days" when follow-up spectroscopy was done on red stars identified in colourmagnitude diagrams. This led to the discovery of the first known carbon stars 
Table 1: Carbon stars in external galaxies

\begin{tabular}{|c|c|c|c|c|c|c|c|}
\hline Name & $\begin{array}{r}\mathrm{D} \\
(\mathrm{kpc})\end{array}$ & $\begin{array}{r}M_{\mathrm{V}} \\
(\mathrm{mag})\end{array}$ & {$[\mathrm{Fe} / \mathrm{H}]$} & $N_{C}$ & $\begin{array}{l}\text { Area } \\
\left(\mathrm{kpc}^{2}\right)\end{array}$ & $N_{M}$ & Refs. \\
\hline M31 & 770 & -21.2 & 0.0 & 243 & 12 & $5254(?)$ & $1,2,3,4$ \\
\hline Galaxy & & -20.6 & 0.0 & 81 & 1.00 & & \\
\hline M33 & 850 & -19.0 & -0.6 & 15 & 0.20 & $60(3+), 15(5+)$ & 2 \\
\hline LMC & 50 & -18.1 & -0.6 & 1045 & 4.8 & $5200(2+), 1300(5+), 475(6+)$ & 6 \\
\hline NGC 6822 & 540 & -16.4 & -0.8 & 36 & 0.18 & $16(3+), 1(5+)$ & 2 \\
\hline NGC 205 & 770 & -16.4 & -0.85 & 7 & 0.30 & $17(2+)$ & 8 \\
\hline SMC & 63 & -16.2 & -1.0 & $\begin{array}{r}789 \\
1707\end{array}$ & $\begin{array}{l}5.4 \\
12.2\end{array}$ & $1250(2+), 180(5+), 60(6+)$ & $\begin{array}{l}6 \\
7\end{array}$ \\
\hline IC 1613 & 765 & -14.9 & -1.2 & 15 & 0.52 & $6(3+), 0(5+)$ & 2 \\
\hline WLM & 940 & -14.0 & -1.5 & 14 & 0.28 & $6(3+), 0(5+)$ & 2 \\
\hline Sagittarius dw & 24 & -14.0 & -0.85 & 26 & & & $9,10,11$ \\
\hline Fornax & 131 & -13.0 & -1.4 & 104 & 0.44 & $25(2+), 4(5+)$ & 12 \\
\hline Leo I & 270 & -12.0 & -2.0 & 16 & 0.45 & & 13 \\
\hline And I & 810 & -11.7 & -1.45 & 0 & & & 16 \\
\hline And II & 700 & -11.7 & -1.3 & 8 & & $1(?)$ & 15,16 \\
\hline Sculptor $d w$ & 78 & -10.7 & -1.8 & 8 & 0.63 & $40(2+), 20(3+)$ & 13 \\
\hline Leo II & 230 & -10.2 & -2.0 & 6 & 0.59 & & 14 \\
\hline And III & 760 & -10.2 & -2.0 & 0 & & & 16 \\
\hline Sextans & 86 & -10.0 & -2.1 & (0) & & & 17 \\
\hline Phoenix & 420 & -9.9 & -2.0 & 2 & 0.44 & & 18 \\
\hline Carina & 87 & -9.2 & -1.5 & 9 & 0.23 & & 13 \\
\hline Ursa Minor & 69 & -8.9 & -2.5 & 1 & 0.58 & & 13 \\
\hline Draco & 76 & -8.6 & -2.3 & 3 & 0.71 & & 13 \\
\hline NGC 55 & 2150 & -20.0 & -0.6 & 14 & 3.2 & $7(5+)$ & 19 \\
\hline NGC 300 & 2150 & -18.7 & -0.4 & 16 & 3.2 & $23(5+)$ & 20 \\
\hline NGC 2403 & 3180 & -20.3 & 0.0 & 4 & 1.6 & $7(?)$ & 21 \\
\hline
\end{tabular}

in e.g. the Carina dwarf (Cannon et al. 1980), or Fornax (Aaronson \& Mould 1980).

Both the GRISM and the narrow-band filter technique allow for a more systematic search for carbon stars over a large area. The former method aims at identifying the prominent $C_{2}$ band heads at 4737 and $5165 \AA$. An illustration of this can be found in Westerlund (1979). The latter method uses standard $V$ and $I$ filters in combination with two narrow-band filters near 7800 and 8100 $\AA$, which are centered on a CN-band in carbon stars, and on a TiO band in oxygen-rich stars, respectively. In a [78-81] versus $[V-I]$ colour-colour plot, carbon stars and late-type oxygen-rich stars clearly separate. For an illustration see Cook \& Aaronson (1989), or Kerschbaum et al. (1998).

\section{Surveys}

In this section the surveys for carbon stars in external galaxies are described. References to the carbon star surveys are listed in Table 1. Labels in the text ("[1]") refer to these references.

The Magellanic Clouds: I reviewed the subject of AGB stars in the Magellanic Clouds at the "ISO's view on stellar evolution" meeting (Groenewegen 1998) and refer to that publication for more details. In brief, based on [6] and [7] the total estimated number of carbon stars in the LMC is about 11000 and about 3100 in the SMC.

Sagittarius dwarf galaxy: [9] identified 4 carbon stars which are members of the Sagittarius dwarf. Infrared photometry for them are listed in Whitelock et al. (1996). This data was transformed from the SAAO to the CTIO system, and bolometric corrections from Frogel et al. (1980) were used to derive 


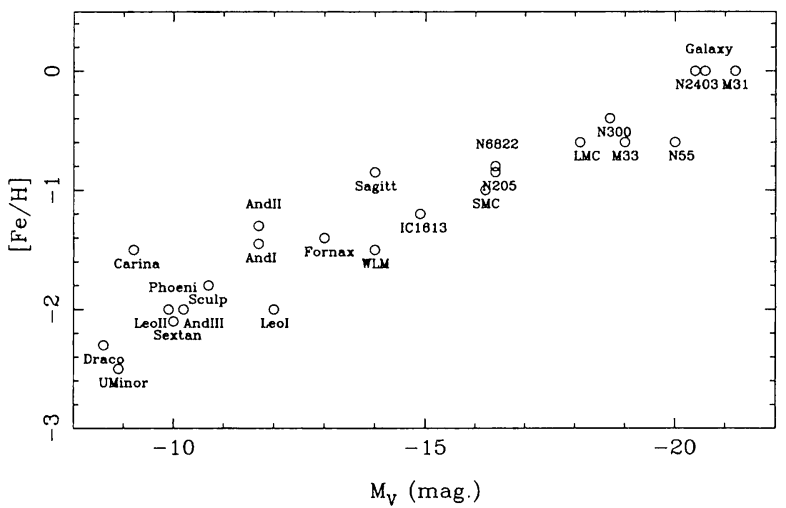

Figure 1. Metallicity versus $\mathrm{M}_{\mathrm{V}}$ in the galaxies.

$m_{\text {bol }}$. [10] suggested another carbon star. Since then, [11] reported on recent work confirming membership for a total of 26 carbon stars, including 8 large- and 7 small-amplitude variables. This marks the discovery of the first carbon-Mira in any of the galactic dwarf spheroidals.

Fornax dwarf galaxy: Fornax has the largest number of C-stars of the galactic dwarf spheroidals and the study of its red population dates back to Demers \& Kunkel (1979), and Aaronson \& Mould (1980). The latest survey ([12]) should be area complete. Published accurate apparent bolometric magnitudes are taken from Frogel et al. (1982) and Aaronson \& Mould (1980) for 15 different stars, based on IR photometry. For three stars in common the average value was taken. [12] mention that they have obtained $J H K$ photometry for $94 \%$ of these stars and find a minimum, mean and maximum absolute bolometric magnitude of $-1.2,-3.7$ and -5.6 , respectively. Frogel et al. (1982), in a smaller field, find $25 \mathrm{C}, 6 \mathrm{M} 2+$ and $0 \mathrm{M} 5+$ stars. Scaling this to the total number of carbon stars I estimate that there should be in total about $25 \mathrm{M} 2+$ and possibly $4 \mathrm{M} 5+$ stars in Fornax.

Leo I dwarf galaxy: The survey by [13] is area complete. It should be noted that Regulus is very close to this galaxy and therefore some carbon stars may have escaped detection. Lee (1993) lists the apparent bolometric magnitudes for 15 objects.

Sculptor dwarf galaxy: The survey by [13] is area complete. Apparent bolometric magnitudes for 2 stars are listed in Frogel et al. (1982). In a smaller area Frogel et al. (1982) found $2 \mathrm{C}-, 10 \mathrm{M} 2+$ and $5 \mathrm{M} 3+$ stars. Scaling this to the total number of carbon stars results in the number for the M-stars in Table 1.

Leo II dwarf galaxy: The survey by [14] is area complete. Apparent bolometric magnitudes are listed in Aaronson \& Mould (1985).

Sextans dwarf galaxy: In the discovery paper of this galaxy by [17] it is mentioned that there is no indication for an AGB in the colour-magnitude diagram. On the other hand, none of the methods mentioned in Sect. 2 was used to actively look for carbon stars.

Phoenix dwarf galaxy: Van de Rydt et al. (1991) provide a detailed colour-magnitude diagram that shows a few candidate AGB stars and covers 

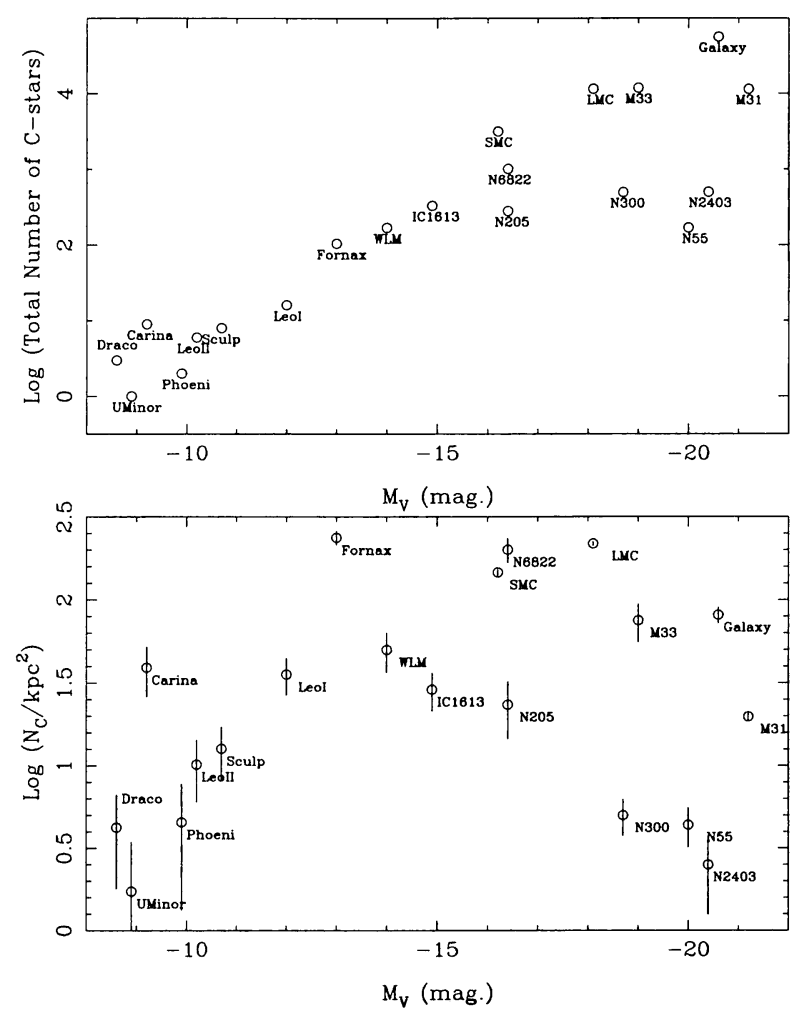

Figure 2. Total number of carbon stars and surface density of carbon stars versus $\mathrm{M}_{\mathrm{V}}$. In the latter diagram no correction for projection effects has been made.

the whole of the galaxy. [18] took spectra of 5 of these candidates and confirmed two as being carbon stars. I have calculated $m_{\text {bol }}$ using the photometry in van de Rydt et al., their adopted reddening and bolometric corrections (BCs) following Bessell \& Wood (1984, hereafter BW84) .

Carina dwarf galaxy: The survey by [13] is area complete. Apparent bolometric magnitudes are available for 6 stars from Mould et al. (1982).

Ursa Minor dwarf galaxy: The survey by [13] is area complete. The apparent bolometric magnitude is listed in Aaronson \& Mould (1985).

Draco dwarf galaxy: The survey by [13] is area complete. Apparent bolometric magnitudes for the 3 stars are listed in Aaronson et al. (1982).

M31: This galaxy was surveyed for carbon stars by $[1,2,3]$ and most extensively by [4]. The latter find a total of $243 \mathrm{C}$ - and 5254 late-M stars (using their colour and magnitude criterion). The $V, I$ data on the individual stars is available on CD-ROM, and was used together with their adopted reddening (depending on the field) and BW84 to obtain $m_{\text {bol }}$. The Brewer et al. survey covers about $2.1 \%$ of the galaxy (this, and similar numbers below is derived from the ratio between survey area and area of the galaxy, as given in the NED database). In a follow-up paper, Brewer et al. (1996) took spectra of some of 

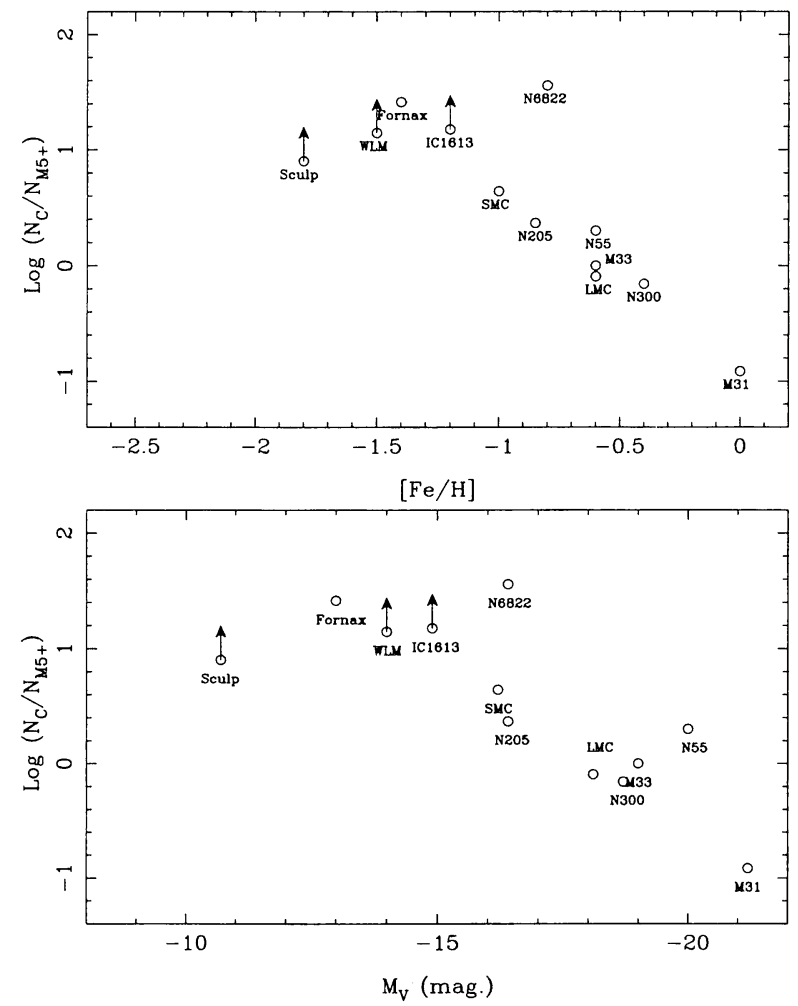

Figure 3. Log of the number ratio of carbon stars to late M-stars versus metallicity and $\mathrm{M}_{\mathrm{V}}$.

the stars previously identified and discovered an S-star, and C-stars enhanced in ${ }^{13} \mathrm{C}$ (J-type) and in Lithium.

M33: The area surveyed by [2] covered about $0.13 \%$ of the galaxy.

NGC 205: This galaxy is a companion of M31. The area surveyed by [8] covered about $2.5 \%$ of the galaxy. Apparent bolometric magnitudes were derived by me from the listed $V, I$ photometry, their adopted reddening, and BCs from BW84.

And I, II, III: And I, II, III are dwarf spheroidal companions to M31. Searches for carbon stars are reported by [15] for And II, and by [16] for all three systems. Aaronson et al. (1985b) found 1 certain, 1 possible C-star and a Mgiant from spectroscopy of red stars. [16] used the narrow-band filter technique to report on no C-stars in And I and III, and 8 candidate C-stars and 3 candidate S-stars in And II, including the one by [15]. The area that was covered in the observations is not quoted by Armandroff.

NGC 6822: The survey by [2] covered about $3.5 \%$ of the galaxy. This galaxy also contains an S-star (Aaronson et al. 1985a)

IC 1613: The survey by [2] covered about $4.5 \%$ of the galaxy.

WLM: The survey by [2] covered about $8.3 \%$ of the galaxy.

NGC 55: This galaxy is in the Sculptor group. [19] surveyed a field covering about $8.3 \%$ of the galaxy. The bolometric magnitude was derived by me from 


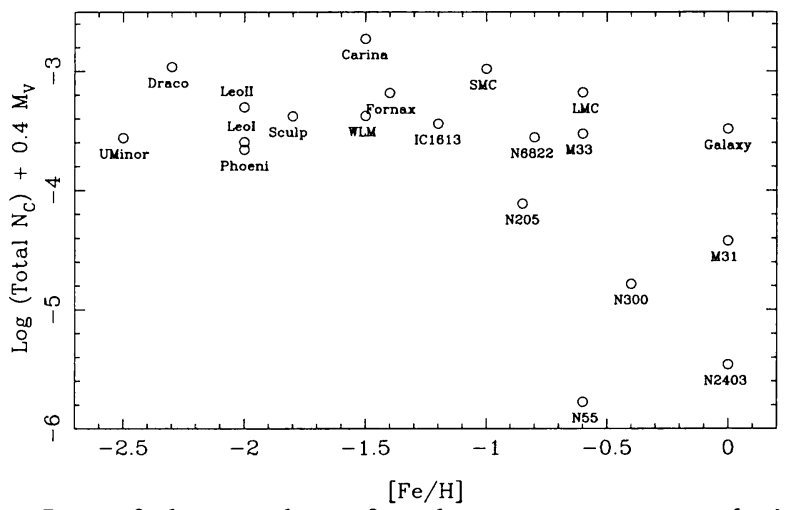

Figure 4. Log of the number of carbon stars over total visual luminosity versus metallicity

the listed $V, I$ photometry, their adopted reddening and the BCs from BW84, except for five stars with a lower limit on $V-I$.

NGC 300: This galaxy is in the Sculptor group. [20] surveyed a field covering about $4 \%$ of the galaxy. The bolometric magnitude was derived by me from the listed $V, I$ photometry, their adopted reddening and the BCs from BW84, except for three stars with a lower limit on $V-I$.

NGC 2403: This galaxy is in the M 81 group, and the most distant galaxy where carbon stars have been identified so far. [21] tentatively identified 4 $\mathrm{C}$ - and $7 \mathrm{M}$-stars, and give the apparent bolometric magnitude based on $R, I$ photometry. Their survey covered about $0.8 \%$ of the galaxy.

Table 1 summarizes the number of known carbon stars in external galaxies. The last three entries are galaxies outside the Local Group. Listed are the adopted distance, absolute visual magnitude, metallicity, number of known carbon stars, the area over which they were found, and the number of late-type M-stars, when known (the symbol ' $3+$ ' meaning stars of spectral type M3 and later, etc.). The entry for the number of carbon stars in our galaxy is the local surface density of TP-AGB stars by Groenewegen et al. (1992).

\section{Discussion}

Fig. 1 shows the well-known correlation between metallicity and absolute visual magnitude. The interpretation being that the more luminous galaxies are also the more massive ones that have had more gas mass available to transform into stars and enrich the interstellar medium.

Fig. 2 shows the number of carbon stars in a galaxy versus $M_{\mathrm{V}}$ represented in two ways. First, the total number of C-stars in a galaxy was estimated by multiplying the known number by the ratio of total surface area of a galaxy to the survey area. As for some galaxies the survey area is less than a few percent, this correction factor can be quite large (and uncertain). To circumvent this, the bottom panel shows the surface density of carbon stars in the particular survey. The drawback of this approach is that it does not take into account the spatial variation of the density of carbon stars within a galaxy. In neither 


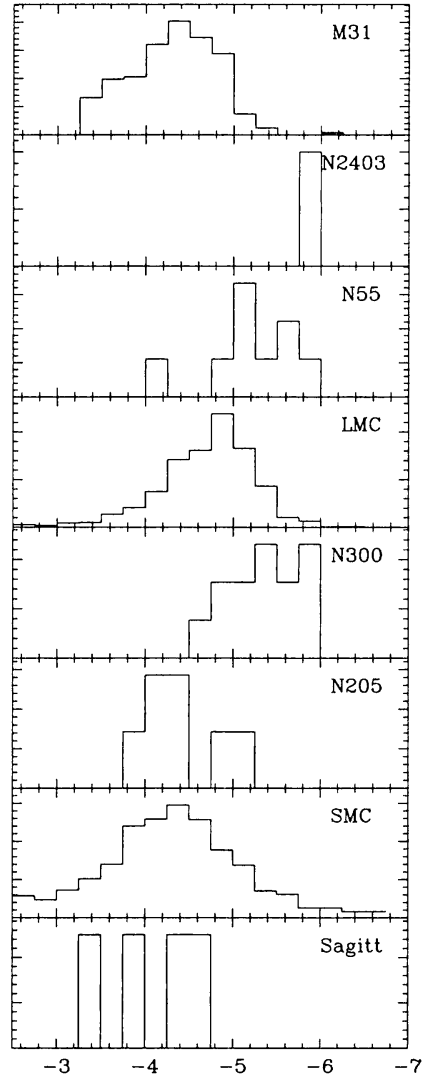

$\mathrm{M}_{\mathrm{bol}}$

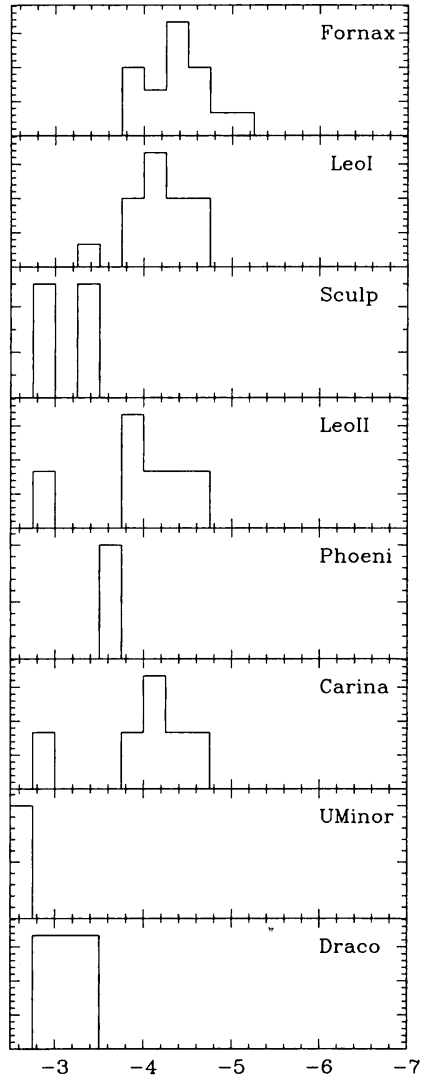

$\mathrm{M}_{\text {bol }}$

Figure 5. Luminosity functions, ordered by decreasing $M_{\mathrm{V}}$. The total number of stars plotted per galaxy is listed in column 6 of Table 2 . The lowest luminosity bin in the SMC and LMC are cumulative.

approach did I correct for the fact that we do not see these galaxies face-on. Some interesting things can be noticed. There is a clear relation between the (estimated) total number of C-stars and $M_{\mathrm{V}}$, and there seems to be a maximum surface density of about $200 \mathrm{kpc}^{-2}$ averaged over a galaxy. In both plots NGC 55,300 and 2403 are clear outliers. These are the most distant galaxies surveyed, and one might suppose that the surveys have been incomplete. For NGC 55 the explanation probably lies as well in the fact that we see this galaxy almost disk-on, and so both the total number as well as the surface density have been underestimated. Reddening within the galactic disk of the galaxy can also play a role. For NGC 2403 the small number of carbon stars lies in the fact that the survey has been incomplete. All $4 \mathrm{C}$-stars have luminosities that are much higher than the average in galaxies for which we know the luminosity function (LF) in more detail. The same is true for NGC 300. A last note of caution is that I did not try to make a distinction between carbon stars on the TP-AGB and lower-luminosity carbon stars. For example, the data for our Galaxy represents 
Table 2. Carbon stars: the luminosity function

\begin{tabular}{lrrrrrr}
\hline Name & $\begin{array}{c}M_{\text {bol }}^{\mathrm{max}} \\
(\mathrm{mag})\end{array}$ & $\begin{array}{r}M_{\text {bol }}^{\mathrm{min}} \\
(\mathrm{mag})\end{array}$ & $\begin{array}{c}M_{\text {bol }}^{\mathrm{mean}} \\
(\mathrm{mag} .)\end{array}$ & $\begin{array}{r}\text { spread } \\
(\mathrm{mag} \text { ) }\end{array}$ & $N_{\text {all }}$ & $N_{\mathbf{M}_{\text {bol }}>-3.5}$ \\
\hline M31 & -6.14 & -3.34 & -4.31 & 0.50 & 243 & 16 \\
NGC 2403 & -5.91 & -5.75 & -5.83 & 0.08 & 4 & 0 \\
NGC 55 & -5.79 & -4.18 & -5.21 & 0.52 & 9 & 0 \\
LMC & -6.30 & -1.25 & -4.70 & 0.55 & 923 & 25 \\
NGC 300 & -5.91 & -4.66 & -5.37 & 0.41 & 13 & 0 \\
NGC 205 & -5.01 & -3.91 & -4.38 & 0.44 & 7 & 0 \\
SMC & -8.17 & -1.62 & -4.32 & 0.81 & 1626 & 227 \\
Sagittarius & -4.61 & -3.29 & -4.08 & 0.60 & 4 & 1 \\
Fornax & -5.22 & -3.81 & -4.35 & 0.37 & 15 & 0 \\
Leo I & -4.68 & -3.38 & -4.17 & 0.32 & 15 & 1 \\
Sculptor & -3.33 & -2.95 & -3.14 & 0.27 & 2 & 2 \\
Leo II & -4.51 & -2.91 & -3.91 & 0.55 & 6 & 1 \\
Phoenix & -3.71 & -3.55 & -3.63 & 0.11 & 2 & 0 \\
Carina & -4.55 & -2.84 & -4.00 & 0.61 & 6 & 1 \\
Ursa Minor & -2.74 & -2.74 & -2.74 & 0.00 & 1 & 1 \\
Draco & -3.45 & -2.75 & -3.10 & 0.35 & 3 & 3 \\
\hline
\end{tabular}

TP-AGB stars only, while on the other hand the SMC is known to contain a large fraction of low-luminosity carbon stars (see later).

Fig. 3 shows the ratio of C- to late M-stars, and it confirms the well known trend with metallicity (Cook et al. 1986; Pritchet et al. 1987). The interpretation is that a star with a lower metallicity needs fewer thermal pulses to turn from an oxygen-rich star into a carbon star.

Fig. 4 shows the ratio of the total estimated number of carbon stars over the visual luminosity of the galaxy (e.g. Aaronson et al. 1983). Most of the galaxies scatter between a value of -3 and -4 , with a few outliers which are the same as noticed in Fig. 2.

Fig. 5 shows the C-stars bolometric LF for the galaxies for which it could be constructed (Sect. 2); for the Magellanic Clouds see Groenewegen (1998) for details. Table 2 lists the maximum, minimum and mean magnitude, as well as the spread, calculated from the rms deviation from the mean. Also listed are the number of stars that went into the calculation and are plotted in Fig. 5, and the number of C-stars that are fainter than $M_{\text {bol }}=-3.5$. The latter is an approximate borderline (which is in fact dependent on metallicity) between TP-AGB and low-luminosity C-stars. The data show that in well populated LF, the mean $M_{\text {bol }}$ is between -4 and -5 . It also shows that the mean in NGC 300 and NGC 2403 is much higher. Unless one would invoke a large uncertainty in the distance or a burst of recent star formation, the most natural explanation lies in the incompleteness of the surveys in these distant galaxies. Finally, the data shows that in the fainter galaxies the mean magnitude increases and that a fair number of C-stars are of the low-luminosity type. This is more clearly seen in Fig. 6 where the mean magnitude is plotted. This indicates a low star formation over the last few Gyrs.

In principle, the overall carbon star LF contains information about the star-formation-rate history from, say, $10 \mathrm{Gyr}$ ago (the low-luminosity C-stars in 

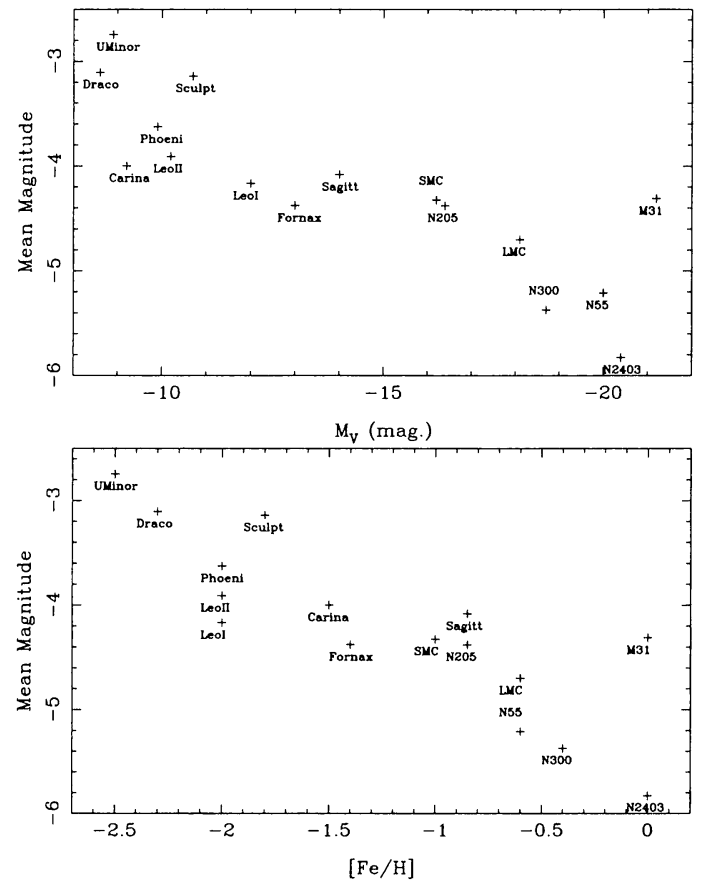

Figure 6. Mean bolometric magnitude of carbon stars versus $M_{V}$ and metallicity.

binaries) to a few-hundred Myr ago (the high luminosity tail of the LF). It is a challenge to theoretical models to use these constraints together with other data to derive the chemical evolution- and star-formation rate history of these galaxies.

\section{References}

Aaronson M., Mould J., 1980, ApJ 240, 804

Aaronson M., Mould J., 1985, ApJ 290, 191

Aaronson M., Liebert J., Stocke J., 1982, ApJ 254, 507

Aaronson M., Mould J., Cook K.H., 1985a, ApJ 291, L41

Aaronson M., Olszewski E.W., Hodge P.W., 1983, ApJ 267, 271

Aaronson M., Gordon G., Mould J., Olszewski E.W., Suntzeff N., 1985b, ApJ 296, L7

Aaronson M., Da Costa G.A, Hartigan P., Mould J.R., Norris J., Stockman H.S., 1984, ApJ 277, L9

Armandroff T.E., 1994, in "Dwarf galaxies", eds. G. Meylan and P. Prugniel, ESO, Garching, p. 211

Azzopardi M., Lequeux J., Westerlund B.E., 1985, A\&A 144, 388

Azzopardi M., Lequeux J., Westerlund B.E., 1986, A\&A 161, 232 
Azzopardi M., Breysacher J., Muratorio G., Westerlund B.E., 1998, in "Stellar populations in the local group", IAU Symposium 192, in press

Bessell M.S., Wood P.R., 1984, PASP 96, 247 (BW84)

Blanco V.M., McCarthy M.F., 1983, AJ 88, 1442

Brewer J., Richer H.B., Crabtree D.R., 1995, AJ 109, 2480

Brewer J., Richer H.B., Crabtree D.R., 1996, AJ 112, 491

Cannon R.D., Niss B., Nørgaard-Nielsen H.U., 1980, MNRAS 196, 1P

Cook K.H., Aaronson M., 1989, AJ 97, 923

Cook K.H., Aaronson M., Norris J., 1986, ApJ 305, 634

Da Costa G.S., 1994, in "Dwarf galaxies", eds. G. Meylan and P. Prugniel, ESO, Garching, p. 221

Demers S., Kunkel W.E., 1979, PASP 91, 761

Frogel J.A., Persson S.E. Cohen J.G., 1980, ApJ 239, 495

Frogel J.A., Blanco V.M., McCarthy M.F., Cohen J.G., 1982, ApJ 252, 133

Groenewegen M.A.T., 1998, in "ISO's view on stellar evolution", eds. L. Waters,

C. Waelkens, K. A. van der Hucht and P.A. Zaal, Kluwer, Ap\&SS 255, p. 379

Groenewegen M.A.T., de Jong T., van der Bliek N.S., Slijkhuis S., Willems F.J., 1992, A\&A 253, 150

Hudon J.D., Richer H.B., Pritchet C.J., Crabtree D.R., Christian C.A., Jones J., 1989, AJ 98, 1265

Ibata R.A., Gilmore G., Irwin M.J., 1995, MNRAS 277, 1354

Irwin M.J., Bunclark P.S., Bridgeland M.T., McMahon R.G., 1990, MNRAS $244,16 \mathrm{P}$

Kerschbaum F., Nowotny W., Schultheis M., Hron J., 1998, in "Stellar populations in the local group", IAU Symposium 192, in press

Lee M.G., Freedman W., Mateo M., Thompson I., Roth M., Ruiz M.-T., 1993, AJ 106, 1420

McClure R.D., 1997, PASP 109, 256

Mould J., Cannon R.D., Aaronson M., Frogel J., 1982, ApJ 254, 500

Ng Y.K., Schultheis M., 1997, A\&AS 123, 115

Pritchet C.J., Richer H.B., Schade D., Crabtree D.R., Yee H.K.C., 1987, ApJ 323,79

Rebeirot E., Azzopardi M., Westerlund B.E., 1993, A\&AS 97, 603

Richer H.B., Crabtree D.R., 1985, ApJ 298, L13

Richer H.B., Crabtree D.R., Pritchet C.J., 1984, ApJ 287, 138

Richer H.B., Crabtree D.R., Pritchet C.J., 1990, ApJ 355, 448

Richer H.B., Pritchet C.J., Crabtree D.R., 1985, ApJ 298, 240

van de Rydt F., Demers S., Kunkel W.E., 1991, AJ 102, 130

Westerlund B.E., 1979, ESO Messenger 19, 7

Whitelock P.A., 1998, in "Stellar populations in the local group", IAU Symposium 192, in press

Whitelock P.A., Irwin M., Catchpole R.M., 1996, New Astr. 1, 57 